\title{
Efecto del porcentaje de refuerzo frente al desgaste en compuestos de matriz metálica sinterizados con descarga luminiscente anormal
}

\author{
Sandra Pérez-Velásquez ${ }^{\mathrm{a}, \varpi}$, Yaneth Pineda-Triana $^{\mathrm{a}}$, Yesid Aguilar-Castro $^{\mathrm{b}}$, Enrique Vera-López $^{\mathrm{a}}$ \\ ${ }^{a}$ Universidad Pedagógica y Tecnológica de Colombia, Instituto para la Investigación e Innovación en Ciencia y \\ Tecnología de Materiales (INCITEMA), Avda. Central del Norte 39-115. Tunja, Colombia \\ buniversidad del Valle, Grupo TPMR, C/13 \#100-00, Cali, Colombia \\ Autor para la correspondencia: patricia.perez@uptc.edu.co
}

Enviado: 16 Abril 2015; Aceptado: 9 Octubre 2015; Publicado on-Line: 9 February 2016

\begin{abstract}
RESUMEN: En este estudio se muestra un análisis del comportamiento del coeficiente de desgaste en seco de compuestos de matriz metálica (MMC), base acero inoxidable 316 reforzado con partículas de carburo de titanio (TiC) de acuerdo con la norma ASTM G 99-05 en un tribómetro para la realización de ensayos de desgaste mediante el contacto y rozamiento de dos materiales entre sí, uno en forma de disco y otro en forma de varilla o bola (pin-on-disk). En el estudio se evalúa el efecto que tiene el porcentaje de refuerzo en los MMC fabricados con 3, 6 y 9\% (vol.) de TiC, en muestras compactadas a $800 \mathrm{MPa}$, dando lugar a diferentes valores de tamaño de grano, dureza y densidad, los cuales son sinterizados por medio de descarga luminiscente anormal, a una temperatura de $1200^{\circ} \mathrm{C} \pm 5^{\circ} \mathrm{C}$, con atmósfera de protección de $\mathrm{H}_{2}-\mathrm{N}_{2}$ y tiempo de permanencia de 30 minutos. De acuerdo a los resultados obtenidos se concluye que la mejor condición de fabricación de los MMC, se consigue cuando la mezcla contiene $6 \%$ de TiC, alcanzando el menor tamaño de grano, la mayor dureza y el más bajo coeficiente de fricción. En este sentido, se observó que la incorporación de partículas de cerámica (TiC) en matriz de acero austenítico (316) presentan mejoras significativas en la resistencia al desgaste.
\end{abstract}

PALABRAS ClAVE: Carburo de titanio; Coeficiente de desgaste; Coeficiente de fricción; Compuesto de matriz metálica; Granulometría; Pulvimetalurgia

Citation / Cómo citar este artículo: Pérez-Velásquez, A., Pineda-Triana, Y., Aguilar-Castro, Y., Vera-López, E. (2016) "Efecto del porcentaje de refuerzo frente al desgaste en compuestos de matriz metálica sinterizados con descarga luminiscente anormal”. Rev. Metal. 52(1):e059. doi: http://dx.doi.org/10.3989/revmetalm.059.

\begin{abstract}
Effect of the percentage of reinforcement on the wear in the metal matrix composites sintered with abnormal glow discharge. In this study an analysis of the behavior of dry wear coefficient of a Metal Matrix Composite (MMC) in 316 stainless steel reinforced with particles of titanium carbide (TiC) according to ASTM G 99 standards, in a pin-on-disk test. In this research it is tested the effect of the percentage of reinforcement in the MMC manufactured with 3, 6 y 9\% (vol.) of TiC, in samples compacted at $800 \mathrm{MPa}$, generating different values of grain size, hardness and density, they are sintered with abnormal glow discharge, at a temperature of $1200{ }^{\circ} \mathrm{C} \pm 5^{\circ} \mathrm{C}$, with a protection atmosphere $\mathrm{H}_{2}-\mathrm{N}_{2}$ and a permanence time of 30 minutes. According to the results obtained it is concluded that the best condition for the MMC manufacturing, in relation to the reinforcement percentage, is the one obtained when the mixture contains $6 \%$ of TiC compacted at $800 \mathrm{MPa}$. In these conditions, it was obtained: achieving smaller grain size, the greater hardness and the lowest coefficient of friction. In this respect, it was observed that the incorporation of the ceramic particles (TiC) in a matrix of austenitic steel (316) shows significant improvements in the resistance to the wear.
\end{abstract}

KEYWORDS: Coefficient of friction; Compaction pressure; Metal matrix composite; Powder metallurgy; Titanium carbide; Wear coefficient

Copyright: (C) 2016 CSIC. This is an open-access article distributed under the terms of the Creative Commons Attribution-Non Commercial (by-nc) Spain 3.0 License. 


\section{INTRODUCCIÓN}

La demanda de componentes de acero fabricado por pulvimetalurgia (PM) está aumentando de manera significativa y diferentes aceros PM han encontrado aplicaciones, principalmente en el sector de la automoción para sistemas de motor y de transmisión (Ceschini et al., 2006), tal es el caso del acero austenítico 316, que a pesar de presentar baja resistencia al desgaste es uno de los más empleados en dicho sector (Renedo, 2010; Sheikhzadeh y Sanjabi, 2012; Onuoha et al., 2013). Mediante PM se han obtenido materiales compuestos que presentan una buena distribución de las partículas para los diferentes contenidos de refuerzo que proporciona a los materiales unas propiedades mecánicas y estructurales aceptables (Amigó et al., 2007). Por estas razones la tecnología PM fue seleccionada como el método de procesamiento en este trabajo, para la producción de compuestos de matriz metálica (MMC) reforzados con partículas, ya que actualmente éstos han atraído la atención debido a su alta resistencia al desgaste, resistencia al calor y estabilidad térmica (Srivastava y Das, 2010; Sun et al., 2010), como también, a las ventajas que ocasiona la incorporación de una fase dura en una fase de metal a través de la mezcla de polvos. Las partículas de refuerzo utilizadas en la fabricación de los MMC, generalmente, son óxidos $\left(\mathrm{Al}_{2} \mathrm{O}_{3}, \mathrm{ZrO}_{3}\right)$, carburos ( $\left.\mathrm{TiC}, \mathrm{WC}\right)$ y boruros $\left(\mathrm{TiB}_{2}, \mathrm{CrB}_{2}\right)$. Entre estos refuerzos, el TiC es un firme candidato para ser utilizado, debido a su elevada dureza, alto punto de fusión y buena estabilidad termodinámica que lo hace apto para reforzar matrices de hierro y de acero (Xinhong et al., 2006; Sun et al., 2010; Zhong et al., 2011). Por lo tanto, se espera que el material compuesto resultante presente un mejor comportamiento al desgaste (Doğan et al., 1999).

Teniendo en cuenta que el desgaste de los materiales es uno de los principales problemas en la industria, que afecta en gran parte a los sectores de producción (Abenojar et al., 2003), el objetivo del presente trabajo es estudiar el comportamiento frente al desgaste del MMC preparado con diferentes porcentajes de refuerzo: 3, 6 y 9\% (vol) de TiC, con los que se obtendrán distintos valores en las variables de: tamaño de grano, dureza y densidad. De esta forma se determinarán los parámetros que proporcionen el mejor comportamiento de los MMC, garantizando que la incorporación de partículas de cerámica (TiC) en matrices de acero austenítico (316) pueda conducir a una mejora en la resistencia al desgaste.

\section{MATERIALES Y MÉTODOS}

\subsection{Materiales}

El compuesto de matriz metálica es manufacturado por PM convencional, empleando polvos de acero inoxidable austenítico 316 como matriz metálica,
TABla 1. Composición química del acero 316 (\% peso)

\begin{tabular}{ccccccc}
\hline $\mathbf{C r}$ & $\mathbf{N i}$ & $\mathbf{M o}$ & $\mathbf{M n}$ & $\mathbf{S i}$ & $\mathbf{C}$ & $\mathbf{F e}$ \\
\hline 17 & 12 & 2,5 & 2 & 1 & 0,08 & equilibrio \\
\hline
\end{tabular}

con la composición química mostrada en la Tabla 1 , y carburo de titanio $\mathrm{TiC}(99,95 \%$ de pureza) como refuerzo, con tamaños medio de partícula para el acero de 106, $140 \mu \mathrm{m}$ y para el TiC de 4,056 $\mu \mathrm{m}$. En la Fig. 1, se muestra la morfología del acero, que es la típica del proceso de atomización en agua, que le imprime características como forma irregular que los hace adecuados para procesos de compactación. Así mismo, se observa que las partículas de TiC exhiben forma poligonal y simétrica con presencia de ángulos agudos que permiten el anclaje del refuerzo en la matriz, lo que puede generar una adecuada consolidación del compuesto durante el proceso de sinterización.

\subsection{Método}

Se usaron tres cantidades diferentes de refuerzo: 3, 6 y $9 \%$ (vol.). La mezcla se realiza en un molino planetario marca RESTCH que consta de dos jarras de acero inoxidable con bolas del mismo material de $10 \mathrm{~mm}$ de diámetro con una relación $4: 1$, durante 80 minutos a $180 \mathrm{rpm}$. La homogeneidad de las mezclas es evaluada por microscopía electrónica de barrido.

Posteriormente las muestras son compactadas uniaxialmente a $800 \mathrm{MPa}$, presión con la que se alcanzó la mayor densidad de la preforma, empleándose la prensa hidráulica marca ELE International de $1000 \mathrm{KN}$ de capacidad, obteniendo preformados en verde con dimensiones aproximadas de $12 \mathrm{~mm}$ de diámetro y $4 \mathrm{~mm}$ de espesor.

Después del prensado, las muestras en verde se sinterizan por medio de la generación de una descarga luminiscente anormal de corriente directa (Fig. 2), en una atmósfera de $\mathrm{H}_{2}-\mathrm{N}_{2}$, bajo los siguientes parámetros de operación: presión 3 Torr, voltaje $534 \mathrm{~V}$, corriente de $160 \mathrm{~mA}$ y velocidad de calentamiento de $100{ }^{\circ} \mathrm{C} \mathrm{min}^{-1}$, condiciones con las que se alcanza la temperatura de trabajo $\left(1200{ }^{\circ} \mathrm{C} \pm 5^{\circ} \mathrm{C}\right)$, con una permanencia de 30 minutos, garantizando la presencia de un plasma estable. El enfriamiento se realiza bajo las mismas condiciones en las que fue realizado el tratamiento de las muestras en la descarga luminiscente (Srivastava and Das, 2010).

Al emplear diferentes condiciones de fabricación variando el porcentaje de refuerzo es necesario evaluar: el tamaño de grano alcanzado de acuerdo a la norma ASTM E112 (2013), la dureza bajo lo estipulado en la norma ASTM B934 (2010), empleando el microdurómetro digital modelo QV-1000 DM y la densidad a partir del principio de Arquímedes empleando como fluido alcohol isopropílico. 

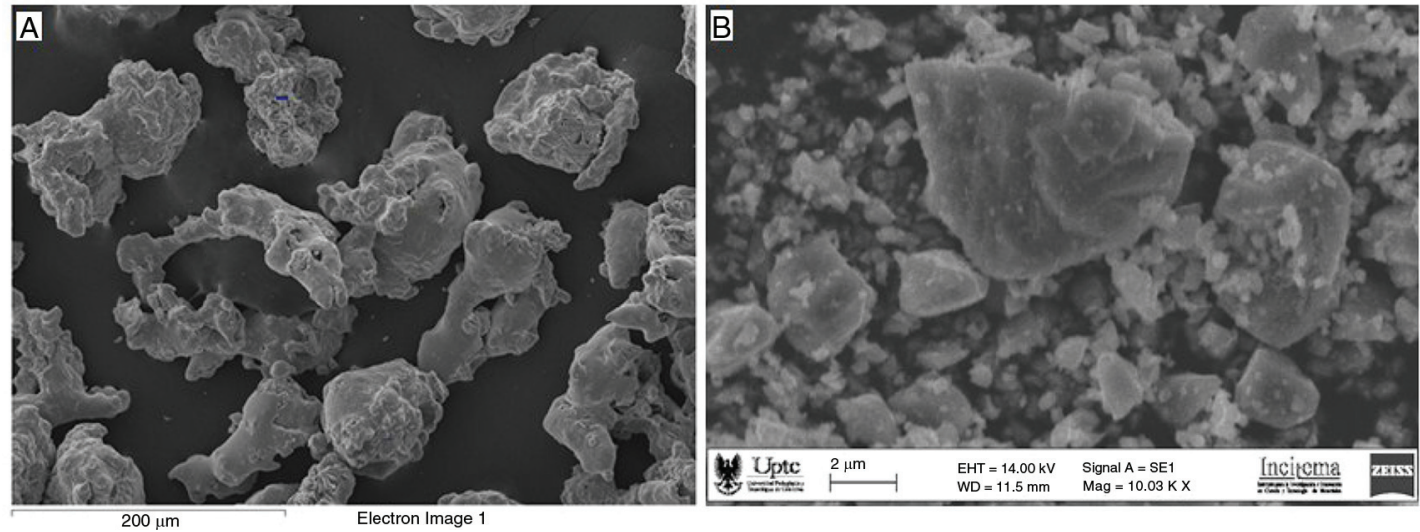

Figura 1. Morfología obtenida con el SEM: a) acero 316 y b) TiC.

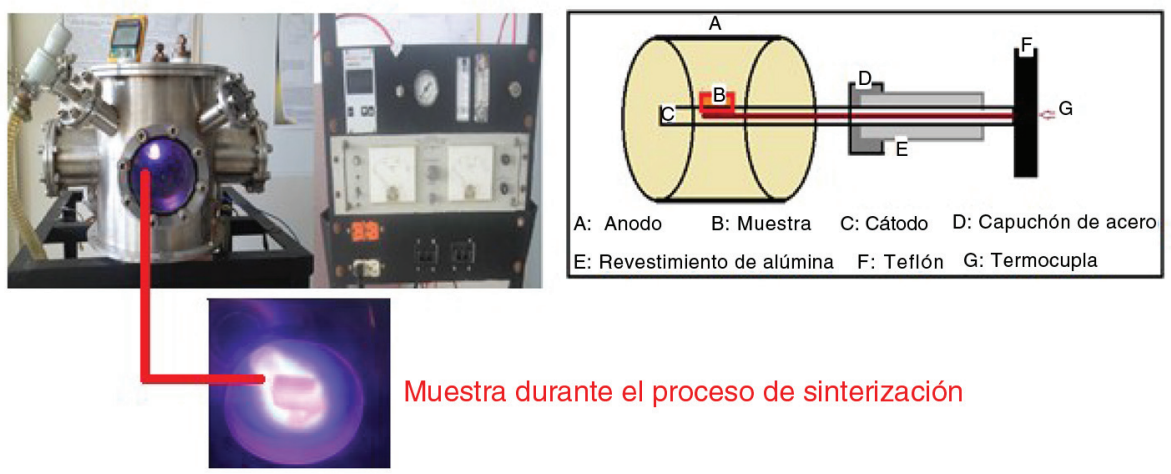

FIGURA 2. Equipo de sinterización mediante descarga luminiscente anormal.

El desgaste se evalúa mediante una prueba de pin-on-disk según la norma ASTM G99-05 (2010) en un tribómetro marca NANOVEA TRB, empleando como contracuerpo un pin de acero de $6 \mathrm{~mm}$ de diámetro y 71,4 RC de dureza. Las pruebas se llevan a cabo a temperatura ambiente y sin lubricación. La carga aplicada es de $5 \mathrm{~N}$, con velocidad de deslizamiento de $0,1 \mathrm{~m} \mathrm{~s}^{-1}$, diámetro de la huella $3,5 \mathrm{~mm} \mathrm{y}$ distancia de deslizamiento de $377 \mathrm{~m}$. El coeficiente de fricción se mide de forma continua durante la prueba y el desgaste se determina por el coeficiente de desgaste $\mathrm{k}$, conforme la Ec. (1):

$$
\mathrm{k}=\mathrm{PPV} /(\mathrm{N} * \mathrm{~m})
$$

en la que el coeficiente de degaste (k) se expresa en $\mathrm{m}^{2} \mathrm{~N}^{-1}$; PPV es la pérdida de volumen del material, expresada en $\mathrm{m}^{3} ; \mathrm{N}$ es la carga aplicada expresada en $\mathrm{N}$ y $\mathrm{m}$ es la distancia de deslizamiento expresada en metros, todo ello, para una humedad relativa $<30 \%$. Con los resultados obtenidos se evalúa el efecto que tiene el porcentaje de refuerzo frente al desgaste producido en los MMC.

\section{RESULTADOS Y DISCUSIÓN}

Indudablemente la industria pulvimetalúrgica ha despertado gran interés por las ventajas competitivas que este proceso de manufactura exhibe en relación con otras técnicas de conformado, tal como lo demostraron Torralba y Campos (2014), concluyendo que el menor consumo de energía lo presenta la PM, con el máximo rendimiento de utilización de materias primas, comparado con la fundición, la extrusión en frío o caliente, la forja y el mecanizado. Sin embargo, para tener éxito en la fabricación del MMC se debe tener estricto control del proceso de sinterización, del tamaño de grano, dureza y densidad.

\subsection{Proceso de sinterización}

La elección correcta del proceso de sinterización es muy importante, hay que utilizar las condiciones de temperatura, tiempo y atmósfera adecuadas para obtener la mejor calidad final de las piezas (Iglesias et al., 2014). Es de resaltar que cuando las muestras 
son sinterizadas mediante métodos convencionales éstas presentan un amplio rango de distribución de tamaños de granos y gran heterogeneidad morfológica, con presencia de mayores tensiones internas que generan mayor contenido de microgrietas en los granos del material que conllevan a la disminución de las propiedades mecánicas (Benavente, 2015). En el presente estudio se evidencia que la etapa de sinterización fue realizada exitosamente por medio de la generación de una descarga luminiscente anormal (plasma), técnica reciente que representa disminución en el tiempo requerido para el proceso de sinterización de muestras y un menor consumo de energía, debido a que el calentamiento se realiza en forma directa por el bombardeo de los iones y átomos neutros sobre la superficie del cátodo donde generalmente se localiza la muestra (Guerrero et al., 2011). Las muestras sinterizadas se prepararon mediante metalografía hasta obtener una superficie pulida a espejo, a la que se le realizó microanálisis utilizando microsonda de RX acoplada al microscopio electrónico (Fig. 3 y Fig. 4).

Se aprecia que las muestras están constituidas principalmente por:
- Oxido de cromo: proviene del oxigeno que se va captando durante el proceso de mezclado. (espectro 1).

- Carburo de titanio: se encuentra localizado en los límites de grano. Su proporción varía, dependiendo del porcentaje que se haya adicionado a cada compuesto. Las partículas conservan la morfología regular y angulosa que las caracteriza originalmente. (espectro 2).

- Matriz: constituida por Fe, Cr, Ni, Mo y Si, constituyentes del acero inoxidable austénitico. (espectro 3).

Así se corrobora que el carburo de titanio es un refuerzo químicamente estable que no reacciona con la matriz, tal como lo demostró el estudio realizado por Ni et al. (2011).

\subsection{Tamaño de grano}

Reducir el tamaño de grano es uno de los principales retos de la PM, sin perder las propiedades deseadas en el MMC, lo cual se debe a que éste tiene considerable influencia en las propiedades mecánicas
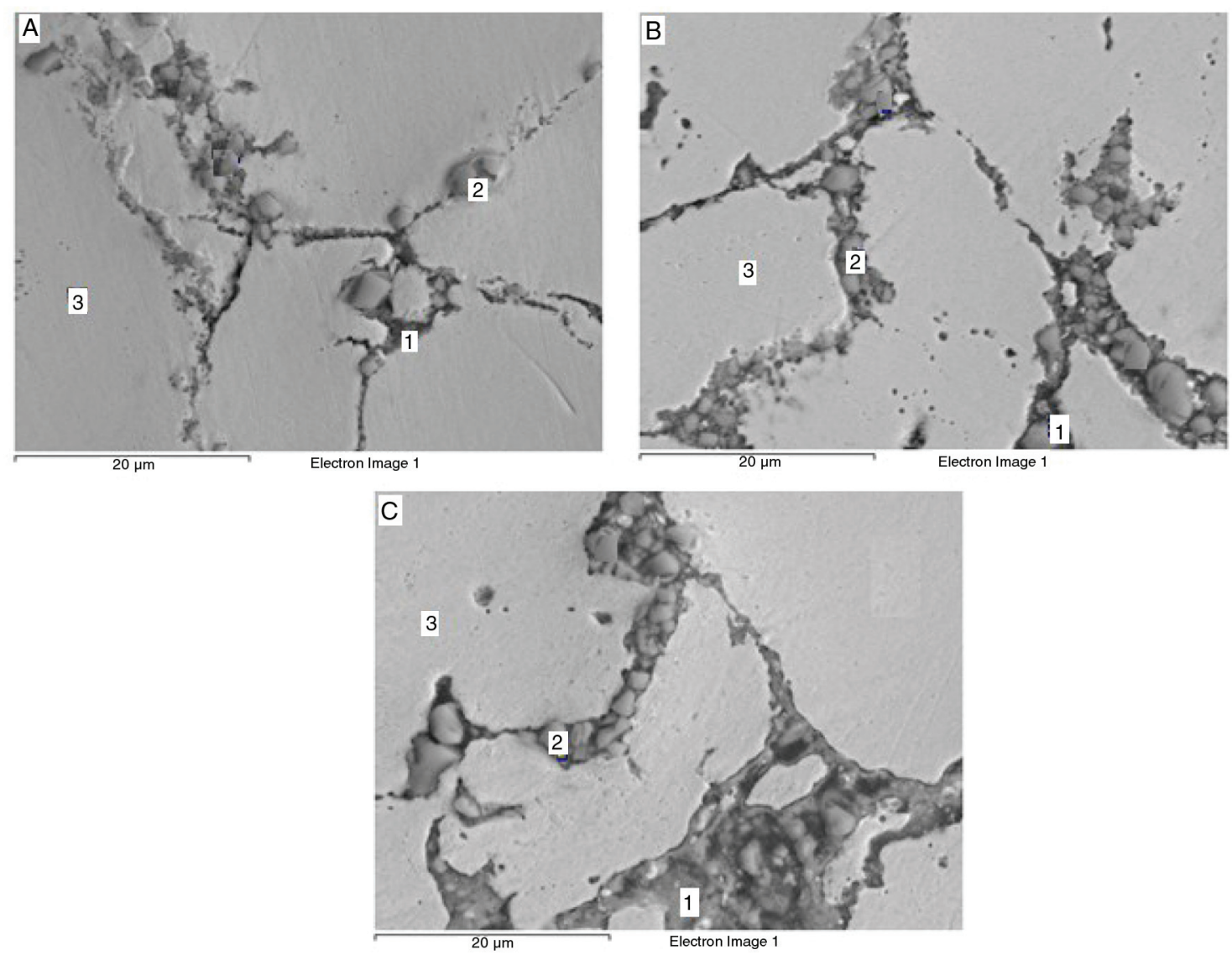

FIgURA 3. Microestructuras obtenidas mediante SEM para los MMC con diferentes porcentajes de refuerzo: a) $3 \% \mathrm{TiC}$, b) $6 \% \mathrm{TiC}$ y c) $9 \% \mathrm{TiC}$. 

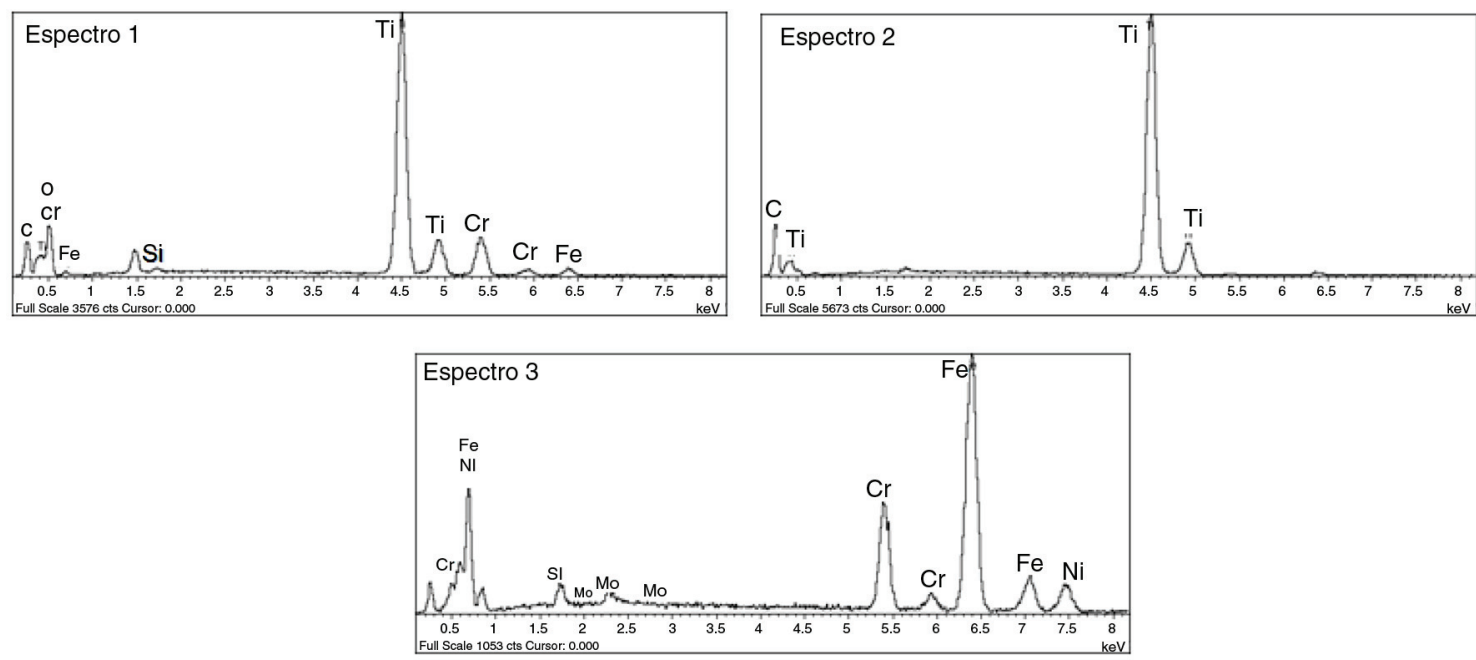

Figura 4. Espectros obtenidos con EDX a tres zonas seleccionadas de los MMC reforzados con 3, 6 y $9 \%$ de TiC.

de los compuestos. El tamaño de grano se determinó mediante el método de líneas horizontales, con una magnificación de la imagen de 100X, conforme a la norma ASTM E112 (2013), en la que se contempla que un índice ASTM grande indica muchos granos, es decir, un tamaño de grano fino, lo que implica altas resistencias. En la Tabla 2 se presenta el número de tamaño de grano de cada uno de los MMC fabricados. Se observa que el menor tamaño de grano se alcanza cuando los MMC están reforzados con $6 \%$ (vol) de TiC. Es de resaltar que los materiales de grano fino serán, por lo tanto, más duros y más resistentes que los materiales de grano grueso ya que éstos tendrán más juntas de grano donde se acumularán más tensiones. Por tanto la adición de TiC ayuda al refinamiento de grano de la matriz austenítica sin formar una nueva fase en la microestructura (Fig. 3), además de mejorar las propiedades mecánicas y resistencia al desgaste (Feng et al., 2012). Así mismo, se evidencia que con el contenido de refuerzo mayor ( $9 \% \mathrm{TiC})$, se incrementa nuevamente el tamaño de grano y el carburo tiende a aglomerarse, lo cual puede ocasionar gran desprendimiento de partículas generando mayor desgaste en el compuesto (Fig. 3c).

Por lo tanto, se considera que el punto de inflexión que presenta el compuesto reforzado con $6 \%$ de

TABla 2. Número de tamaño de grano para los diferentes porcentajes de refuerzo

\begin{tabular}{lcc}
\hline & \multicolumn{2}{c}{ MMC compactados a 800 MPa } \\
\cline { 2 - 3 } \% TiC & Intercepto lineal $(\mu \mathbf{m})$ & Tamaño de Grano ASTM \\
\hline 0 & 59,40 & 4,9 \\
3 & 48,10 & 5,5 \\
6 & 46,10 & 5,6 \\
9 & 51,30 & 5,3 \\
\hline
\end{tabular}

TiC se atribuye a la adecuada homogenización que se alcanza con este porcentaje, generando el menor tamaño de partícula cuando los polvos son sometidos al proceso de mezclado en el que las partículas de TiC se anclan mecánicamente en la superficie de la matriz. Este hecho hace que el material pierda continuidad y que con el permanente sometimiento a esfuerzos cíclicos (golpes de los cuerpos moledores), se generen fisuras internas que derivan en el seccionamiento del material y por ende en la reducción de su tamaño.

\subsection{Dureza}

Para corroborar el efecto que el tamaño de grano tiene sobre la dureza se evaluó cada una de las muestras, evidenciándose que esta propiedad mecánica es inversamente proporcional al tamaño de grano (Tabla 2 y Fig. 5), presentando el mejor comportamiento el MMC reforzado con $6 \%$ de TiC, cuyo tamaño de grano fue el menor. Esto concuerda con el estudio realizado por Xinhong et al. (2006), donde relacionan que al aumentar la fracción de volumen del TiC incrementa la dureza del compuesto, además de obtener el menor desgaste. Sin embargo, al aumentar la fracción de volumen de las partículas de TiC éstas pueden limitar la deformación plástica que la matriz puede absorber lo que conduce a que se produzca fractura y desprendimiento de partículas (Doğan et al., 1999).

\subsection{Densidad}

Las propiedades de un material PM, se producen en gran parte, a través de la mejora del nivel de densidad que se alcance en los compuestos, la cual depende principalmente de la presión de compactación (Gómez et al., 1998). En el presente estudio 


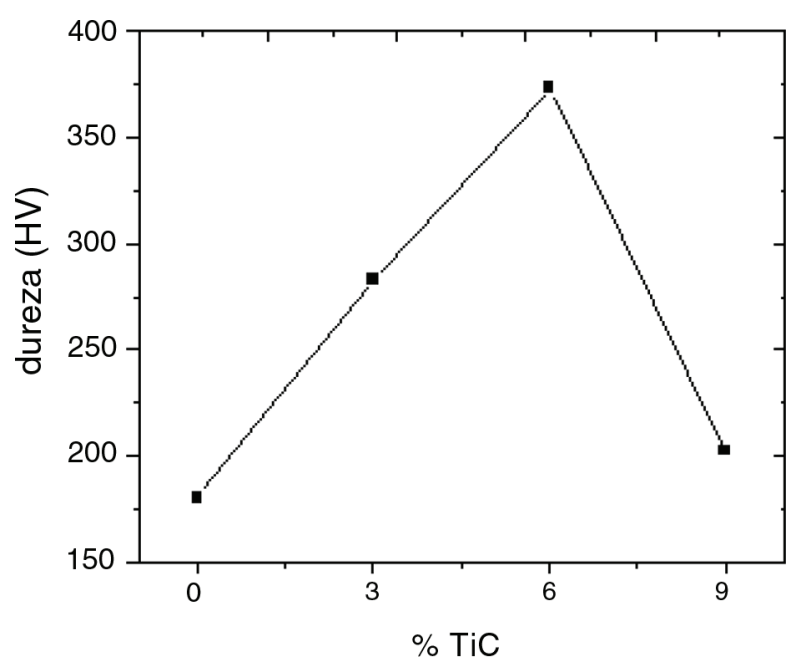

FIGURA 5. Efecto del porcentaje de refuerzo en la dureza.

se analizó la variación de la densidad en las muestras mediante el principio de Arquímedes. El comportamiento presentado se aprecia en la Fig. 6, en la que se muestra que al reforzar el acero inoxidable con el carburo de titanio, se produce una disminución de la densidad, debido al menor peso específico de estos materiales, tal como se evidenció en el estudio realizado por Velasco et al. (2003). La densidad relativa se mantiene alrededor del $82 \%$ en todos los materiales excepto para el compuesto que contiene $9 \%$ de $\mathrm{TiC}$, cuya densidad relativa corresponde al $76 \%$, con lo que se evidencia que la reducción del tamaño de grano permite obtener densidades más altas.

\subsection{Coeficiente de fricción y de desgaste}

En los materiales manufacturados con la técnica de pulvimetalurgia es importante analizar diferentes condiciones de fabricación que conlleven a obtener una mejor respuesta del compuesto en servicio. De acuerdo a lo anterior se evidencia que el porcentaje de refuerzo sí tiene efecto sobre el

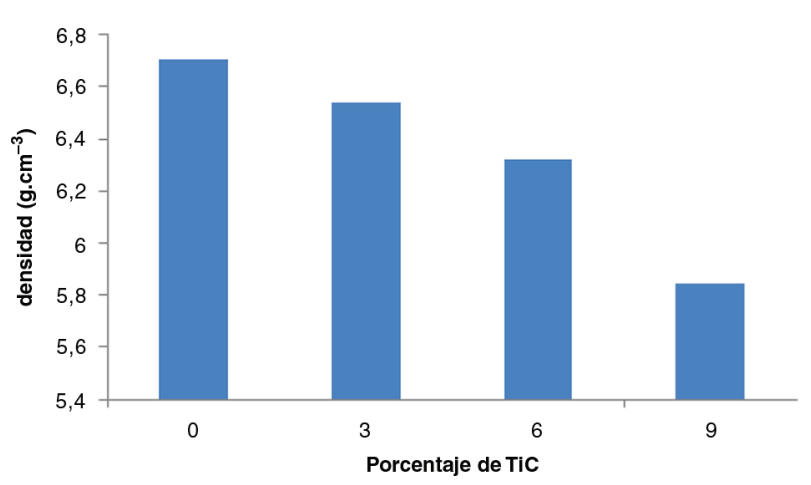

Figura 6. Efecto del porcentaje de refuerzo en la densidad. comportamiento frente al desgaste. Al analizar los valores de los coeficientes de fricción que son obtenidos del tribómetro pin-on-disk durante el ensayo de deslizamiento en seco (Fig. 7), se observa que el menor coeficiente de fricción lo presenta el MMC que contiene $6 \%$ de TiC. Es de resaltar que dicho compuesto es el que exhibe el tamaño de grano más fino y la mayor dureza, lo que puede garantizar que exista una buena interacción entre la matriz y el refuerzo, como se demuestra en la respuesta alcanzada por este mismo compuesto al obtener el menor desgaste (Fig. 7). Así mismo, se aprecia que al aumentar el porcentaje de refuerzo se incrementa el coeficiente de fricción y por lo tanto aumenta el desgaste, por el mayor desprendimiento de partículas, ocasionado por la baja deformación plástica que la matriz puede absorber (Doğan et al., 1999) y por la fragilidad propia del cerámico utilizado como refuerzo. Este comportamiento es muy similar al presentado por el acero sin refuerzo, lo que indica su baja resistencia al desgaste.

El análisis de la huella dejada en los MMC producto de la prueba de desgaste se tomó a una magnificación de 200 aumentos con el método de electrones secundarios y con voltaje de aceleración de $20 \mathrm{kV}$. En la Fig. 8, se indica con una flecha la dirección de deslizamiento, se aprecia que en todos los casos el desgaste que se presenta es de tipo abrasivo, (señalados en la figura con el número 1). La norma ASTM Vol. 03.02 (2013) define este tipo de desgaste como la pérdida de masa resultante de la interacción entre partículas o asperezas duras que son forzadas contra una superficie y se mueven a lo largo de ella.

En la Fig. 8a, se observa que la superficie de la huella es la típica del desgaste abrasivo. A pesar de que este material presenta la mayor densificación no es una condición que contribuya a obtener comportamientos en servicio ideales donde su resistencia ante el desgaste sea la esperada, demostrando que

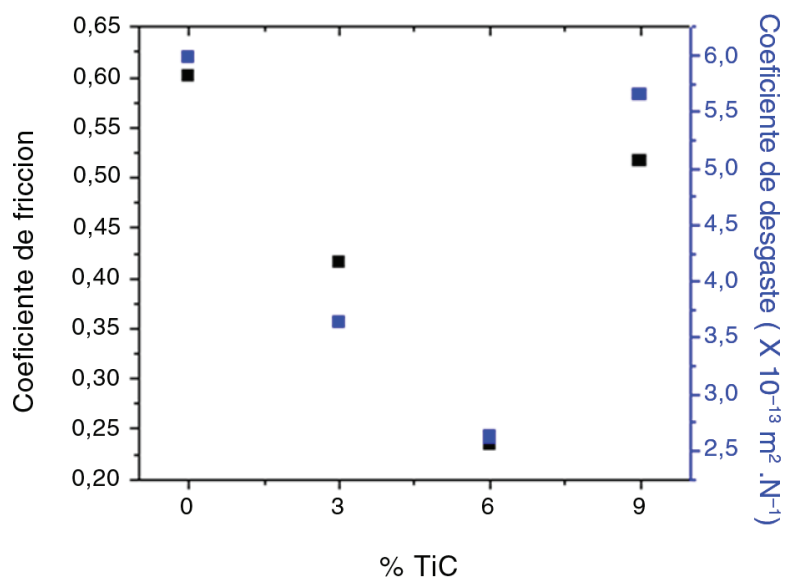

Figura 7. Efecto del porcentaje de refuerzo en el coeficiente de fricción y de desgaste. 

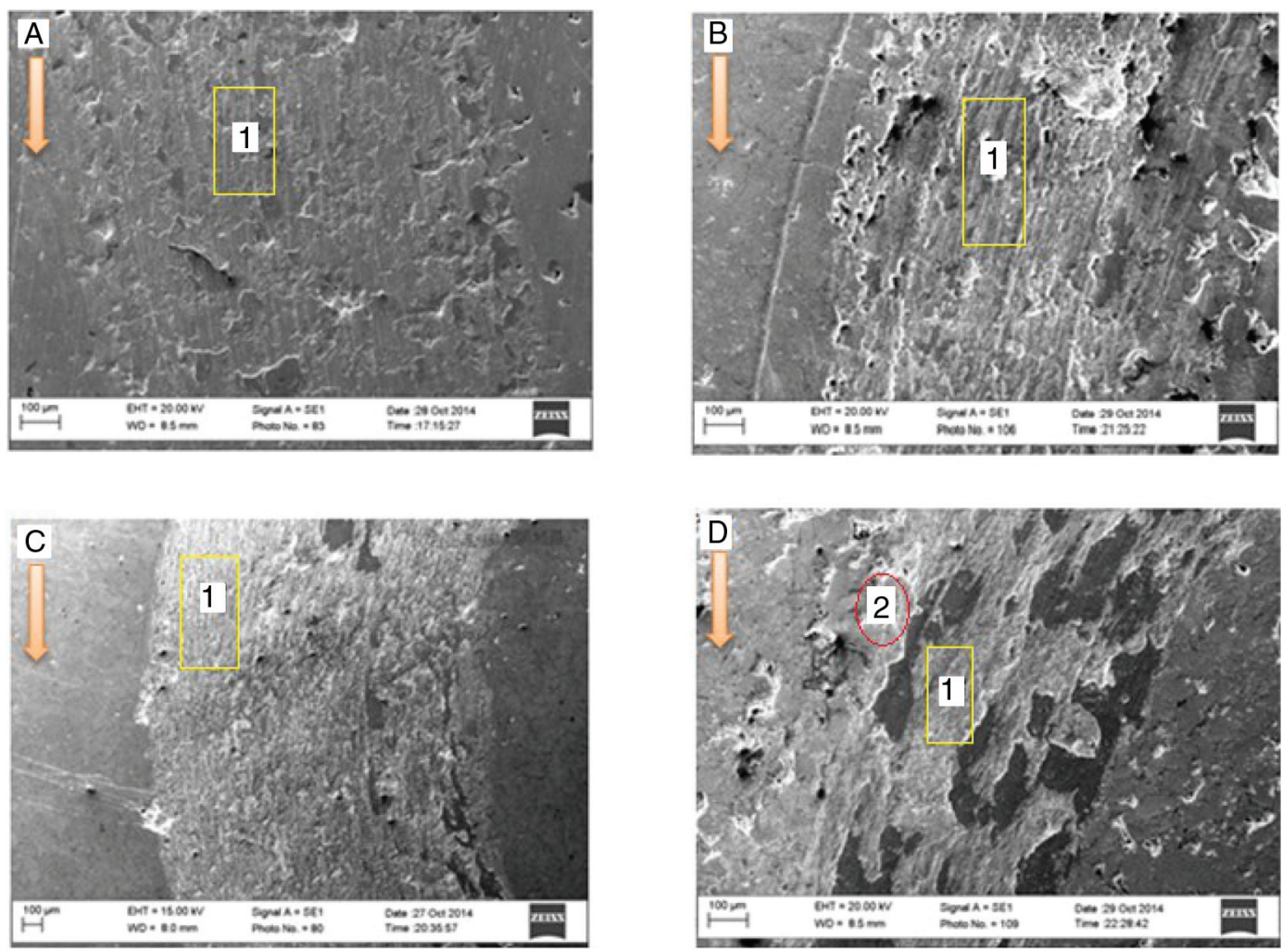

FIGURA 8. Microestructuras obtenidas mediante SEM para MMC con diferentes porcentajes de refuerzo: a) $0 \% \mathrm{TiC}$, b) $3 \% \mathrm{TiC}$, c) $6 \% \mathrm{TiC}$ y d) $9 \% \mathrm{TiC}$.

es necesario la incorporación de partículas que actúen como refuerzo en la matriz de acero austenítico con el fin de obtener una mejor respuesta ante el desgaste. Así mismo, se evidencia que los MMC exhiben una huella más irregular por la presencia del carburo de titanio (Fig. 8b, c y d), ya que se presenta desprendimiento de partículas, siendo mayor cuando el compuesto incrementa el porcentaje de refuerzo a $9 \%$ (señalado con el número 2). Con esta mezcla la densificación y la dureza alcanzada no permite que exista una buena interacción entre la matriz y el refuerzo, ocasionando que el desgaste abrasivo que presenta el compuesto se deba a la presencia de tres cuerpos. Es de resaltar que el compuesto que contiene $6 \%$ de TiC (Fig. 8c) presenta las mejores condiciones de producción alcanzando la mayor dureza y el más bajo coeficientes de fricción, obteniéndose un buen comportamiento frente al desgaste.

\section{CONCLUSIONES}

- A partir de esta investigación se demuestra que mediante la generación del plasma producido por la descarga luminiscente se logra sinterizar material compuesto manufacturado por pulvimetalurgia, alcanzando la temperatura de trabajo en un menor tiempo.
- El acero PM exhibe el mayor desgaste con un alto coeficiente de fricción y baja dureza, demostrando que la mezcla de partículas cerámicas en una matriz austenítica permite mejorar las propiedades de desgaste de todo el sistema. Lo anterior es válido para los tres porcentajes de refuerzo empleados en la fabricación de los MMC. Se evidencia que el tamaño de grano, densidad y dureza alcanzada con el 6\% (vol) de $\mathrm{TiC}$, garantiza que los MMC presenten el más bajo coeficiente de fricción $(0,2355)$ y el menor coeficiente de desgaste $\left(2,62 \times 10^{-13} \mathrm{~m}^{2} \mathrm{~N}^{-1}\right)$.

Así se determina que el tipo de desgaste presente en todas las muestras corresponde a desgaste abrasivo, en el que se evidencia desprendimiento de material y presencia de huellas características de este tipo de desgaste, siendo más visibles en los contenidos altos de refuerzo $(9 \% \mathrm{TiC})$, ocasionado porque el compuesto experimenta desgaste abrasivo de tres cuerpos.

\section{REFERENCIAS}

Abenojar, J., Velasco, F., Bautista, A., Campos, M. (2003). Atmosphere influence in sintering process of stainless steels matrix composites reinforced with hard particles. Compos. Sci. Technol. 63 (1), 69-79. http://dx.doi.org/ 10.1016/S0266-3538(02)00179-3. 
Amigó, V., Romero, F., Salvador, M.D., Busquets, D. (2007). Reactividad matriz-refuerzo en compuestos de matriz de titanio pulvimetalurgico. Rev. Metal. 43 (6), 434 447. http://dx.doi.org/10.3989/revmetalm.2007.v43.i6.

ASTM B934 (2010). Standard Test Method for Effective Case Depth of Ferrous Powder Metallurgy (P/M) Parts Using Microindentation Hardness Measurements, ASTM International, West Conshohocken, USA.

ASTM G99-05 (2010). Standard TestMethod for Wear Testing with a Pin on Disk Apparatus, ASTM International, West Conshohocken, PA, California, USA.

ASTM E112 (2013). Standard Test Methods for Determining Average Grain Size, ASTM International, West Conshohocken, PA, USA.

ASTM Vol. 03.02 (2013). Corrosion of Metals; Wear and Erosion, West Conshohocken, PA, USA.

Benavente, R. (2015). Desarrollo de materiales cerámicos avanzados con altas prestaciones mediante técnicas no convencionales de sinterización: microondas. Tesis Doctoral, Universidad Politécnica de Valencia (UPV), p. 243.

Ceschini, L., Palombarini, G., Sambogna, G., Firrao, D., Scavino, G., Ubertalli, G. (2006). Friction and wear behaviour of sintered steels submitted to sliding and abrasion tests. Tribol. Int. 39 (8), 748-755. http://dx.doi.org/10.1016/j. triboint.2005.07.003.

Doğan, Ö., Hawk, J., Tylczak, J., Wilson, R., Govier, R. (1999). Wear of titanium carbide reinforced metal matrix composites. Wear 225-229, 758-769. http://dx.doi.org/ 10.1016/S0043-1648(99)00030-7.

Feng, S.-R., Tang, H.-B., Zhang, S.-Q., Wang, H.-M. (2012). Microstructure and wear resistance of laser clad $\mathrm{TiB}-\mathrm{TiC} / \mathrm{TiNi}-\mathrm{Ti}_{2} \mathrm{Ni}$ intermetallic coating on titanium alloy. T. Nonferr. Metal. Soc. 22 (7), 1667-1673. http:// dx.doi.org/10.1016/S1003-6326(11)61371-X

Gómez, F., Caballero, J.M., Herrera, E.J. (1998). Algunas observaciones sobre la sinterización del acero austenítico 316L en atmósfera de argón. Rev. Metal. 34 ( ${ }^{\circ}$ Extra), 267-270. http://dx.doi.org/10.3989/revmetalm.1998.v34. iExtra. 751 .

Guerrero, U., Parra, C., Sarmiento, A. (2011). Procesamiento en descarga luminiscente de la perovskita $\mathrm{YBa}_{2} \mathrm{Cu}_{3} \mathrm{O}_{7-\mathrm{d}}$. DYNA 78 (169), 201-205.

Iglesias, F., Martínez, C., Cotes, M.T., Ruiz, J.M., García, L.E., Corpas, F.A. (2014). Influencia de la atmósfera de sinterización en las propiedades mecánicas de los aceros P/M AISI 430L. Rev. Metal. 50 (1), e008. http://dx.doi. org/10.3989/revmetalm.008.

Ni, Z., Sun, Y., Xue, F., Bai, J., Lu, Y. (2011). Microstructure and properties of austenitic stainless steel reinforced with in situ TiC particulate. Mater. Design. 32 (3), 1462-1467. http://dx.doi.org/10.1016/j.matdes.2010.08.047.

Onuoha, C., Kipouros, G., Farhat, Z., Plucknett, K. (2013). The reciprocating wear behaviour of $\mathrm{TiC}-304 \mathrm{~L}$ stainless steel composites prepared by melt infiltration. Wear 303 (1-2), 321-333. http://dx.doi.org/10.1016/j.wear.2013.03.040

Renedo, J. (2010). Comportamiento al desgaste por deslizamiento en aceros inoxidables: ferrítico, austenítico, dúplex y martensítico. Proyecto final de carrera, Universidad Politécnica de Catalunya (UPC).

Sheikhzadeh, M., Sanjabi, S. (2012). Structural characterization of stainless steel/TiC nanocomposites produced by highenergy ball-milling method at different milling times. Mater. Design. 39, 366-372. http://dx.doi.org/10.1016/j. matdes.2012.02.011.

Srivastava, A.K., Das, K. (2010). The abrasive wear resistance of TiC and (Ti,W) C-reinforced Fe-17Mn austenitic steel matrix composites. Tribol. Int. 43 (5-6), 944-950. http:/l dx.doi.org/10.1016/j.triboint.2009.12.057.

Sun, G.J., Wu, S.J., Su, G.C. (2010). Research on impact wear resistance of in situ reaction $\mathrm{TiCp} / \mathrm{Fe}$ composite. Wear 269 (3-4), 285-290. http://dx.doi.org/10.1016/j.wear.2010. 04.011 .

Torralba, J.M., Campos, M. (2014). Toward High Performance in Powder Metallurgy. Rev. Metal. 50 (2), e017. http://dx.doi. org/10.3989/revmetalm.017.

Velasco, F., Lima, W.M., Antón, N., Abenójar, J., Torralba, J.M. (2003). Effect of intermetallic particles on wear behaviour of stainless steel matrix composites. Tribol. Int. 36, (7), 547-551. http://dx.doi.org/10.1016/S0301-679X(02)00264-5.

Xinhong, W., Zengda, Z., Sili, S., Shiyao, Q. (2006). Microstructure and wear properties of in situ $\mathrm{TiC} / \mathrm{FeCrBSi}$ composite coating prepared by gas tungsten arc welding. Wear 260 (7-8), 705-710. http://dx.doi.org/10.1016/j.wear.2005.03.018.

Zhong, L., Xu, Y., Hojamberdiev, M., Wang, J., Wang, J. (2011). In situ fabrication of titanium carbide particulates-reinforced iron matrix composites. Mater. Design. 32 (7), 3790-3795. http://dx.doi.org/10.1016/j.matdes.2011.03.031. 